\title{
Inference Versus Description: The Issue Of Sampling And Areal Units
}

\author{
Robert C. Larson*
}

The application of statistical techniques to areal data gives rise to some conceptual and methodological problems. In attempts to disclose urban spatial pattern we are often constrained to the use of areal units by the very nature of available data. The use of statistical methods with this type of data is the subject of this comment.

Gould has discussed the inappropriateness of employing inferential techniques when descriptive methods are totally adequate (Gould, 1970:442). Specifically, it cannot be considered appropriate to employ inferential techniques when an entire population, rather than a sample, is studied. In such a case, inferential tests of significance are at best superfluous and may serve only to cloud the description of the population. This is the argument which Meyer has applied specifically to the study of spatial variation among areal units (Meyer, 1972:26-28). When areal units are employed the distinction between geographic sample and population may be unclear. Meyer considered the example of a correlation analysis involving an entire population of census tracts, and he concluded that inferential methods are inappropriate since all tracts are included in the analysis (Meyer, 1972:26-28). However, it is my contention that this conclusion is overly simplistic. In an analysis such as the one Meyer considered we may indeed require inferential techniques.

It would seem that confusion may arise over defining the "unit of interest." The unit of observation need not and often cannot coincide with the unit we may wish to learn about. Meyer recognized the neglect of scale considerations in spatial research (Meyer, 1972:26-28). The units of observation may be of a scale different from the units of interest. If we are indeed interested in patterns and relationships among areal units, then our scale of observation may coincide with our scale of interest (i.e., census tracts). However, it is more likely that we may be interested in the spatial patterns and relationships among individuals (persons, households, housing units, etc). In this case, the scales are not coincident, since our scale of observation is not individuals but census tracts. In such a case the tracts must be considered as tools of the analysis and not the units of interest whose spatial relationships we wish to describe. Clearly, it will be necessary to make inferences from the areal analysis to the case of the individual.

The use of inferential statistics assumes that some form of sampling is

* Department of Ceography and L'rban Studies, California State L'niversity, Los Angeles, California 90032. 
necessary or at least possible. As Curry stated: "Probabilistic analysis beyond description is warranted only when we can conceive of a sample being taken other than the original one" (Curry, 1966:98). It is this sampling issue which is unclear when areal units are employed. Nevertheless, the process of partitioning a region into areal units can and often should be viewed as a sampling procedure. This is the case when we are interested in spatial relationships a mong individuals but employ areal unit data to study these relationships. Furthermore, it is not necessary, as Curry has suggested, for samples to be possible for different times and places. The universe need not be defined in that fashion, as illustrated in the following paragraph.

Consider a hypothetical example of spatial correlation analysis between two variables. Figure 1 illustrates the population of 24 individuals under study. The letter " $\mathrm{H}$ " indicates high levels of both variables, and a letter " $\mathrm{L}$ " indicates low levels of both. The example may appear trivial since the entire population is easily studied. However, a small example serves the purpose of illustration. The computed correlation coefficient for the entire population illustrated in Figure 1 might be $r=1.00$. When the number of individuals is too large for study, or data is not available on an individual basis, areal unit data may be employed. Thus we use a smaller number of areal units to study a large number of individuals. In such a case, the set of areal units may be considered a spatial "aggregate sample." Under normal sampling procedures, each member of the sample is an individual. In an aggregate sample (a set of areal units) each member of the sample is an aggregate of individuals. The hypothetical universe is the set of all possible "aggregate samples" (sets of areal units) of the data.

\begin{tabular}{|cccc|}
\hline H & H & H & H \\
H & H & H & H \\
H & H & H & H \\
L & L & L & L \\
L & L & L & L \\
L & L & L & L \\
\hline
\end{tabular}

Figure 1. The population of individuals. Correlation coefficient approximately equal to 1.00 


\begin{tabular}{|llll|}
\hline $\mathrm{H}$ & $\mathrm{H}$ & $\mathrm{H}$ & $\mathrm{H}$ \\
$\mathrm{H}$ & $\mathrm{H}$ & $\mathrm{H}$ & $\mathrm{H}$ \\
$\mathrm{H}$ & $\mathrm{H}$ & $\mathrm{H}$ & $\mathrm{H}$ \\
\hline $\mathrm{L}$ & $\mathrm{L}$ & $\mathrm{L}$ & $\mathrm{L}$ \\
$\mathrm{L}$ & $\mathrm{L}$ & $\mathrm{L}$ & $\mathrm{L}$ \\
$\mathrm{L}$ & $\mathrm{L}$ & $\mathrm{L}$ & $\mathrm{L}$ \\
\hline
\end{tabular}

Figure 2. $N=2$. Correlation coefficient approximately equal to 1.00 .

We have noted that the correlation coefficient for the population data might approach $r=1.00$, and this is the result we would hope to estimate using areal unit data. Figure 2 represents a possible division of the population into two areal units $(\mathrm{N}=2)$. This aggregate sample yields a correlation coefficient $r$ of approximately 1.00 and therefore successfully estimates the correlation based on population data. In contrast, the population might be

\begin{tabular}{|cc|cc|}
\hline H & H & H & H \\
H & H & H & H \\
H & H & H & H \\
L & L & L & L \\
L & L & L & L \\
L & L & L & L \\
\hline
\end{tabular}

Figure 3. $N=2$. Correlation coefficient approximately equal to 0.00 . 
divided into two areal units as in Figure 3. Here the correlation coefficient approaches $r=0.00$, and this aggregate sample unsuccessfully simulates the results of the population analysis. Clearly there may be a large number of ways to partition a population into $\mathrm{N}$ areal units. While $\mathrm{N}$ may be held constant in each case, the correlation coefficient may vary greatly depending upon the aggregate sample chosen. As in any sampling procedure, the size of the sample ( $\mathrm{N}$, the number of areal units) may be varied, and this may affect the results of the correlation analysis. A comparison of Figures 2 and 4 illustrates this point. Clearly a correlation analysis based on the areal units of Figure 4 where $\mathrm{N}=3$ would yield a coefficient substantially weaker than would be obtained from an analysis based on Figure 2 where $\mathrm{N}=2$. In short, aggregate samples of a population may vary with respect to both sample size and the character of the sample members. Both may affect the analysis.

When we employ inferential tests of statistical significance we are testing to be reasonably certain that a result was not obtained by accident. We have demonstrated that arbitrary decisions concerning areal unit boundaries and the number of areal units can inadvertantly affect the results of a statistical analysis. Thus, since "accidental results" are possible, statistical analysis of areal units may be inferential rather than descriptive. Of course, biases in spatial aggregate samples need not be accidental. The opportunities for the gerrymandering of areal unit boundaries within a city are obvious. The case for the purely descriptive role of statistics assumes areal units which are meaningful entities rather than arbitrary divisions of space and which are "units of interest" as well. When this assumption is met, the argument presented by Meyer becomes valid, and

\begin{tabular}{|llll|}
\hline H & H & H & H \\
H & H & H & H \\
\hline H & H & H & H \\
L & L & L & L \\
\hline L & L & L & L \\
L & L & L & L \\
\hline
\end{tabular}

Figure 4. $N=3$. Correlation coefficient: $0.00<\mathrm{r}<1.00$. 
the analysis is descriptive (Meyer, 1972:26-28). However, the assumption may rarely be met for many of the areal units commonly employed, such as census tracts. Furthermore, when the assumption is not met, inferential methods are warranted.

\section{REFERENCES}

CURRY, LESLIE (1966) "A Note on Spatial Association." Professional Geographer 18:98.

GOULD, PETER (1970) "Is Statistix Inferens the Geographical Name for a Wild Goose?" Economic Geography $46: 2$.
MEYER, DAVID (1972) "Geographical Population Data: Statistical Description Not Statistical Inference." Professional Geographer 24:26-28. 